Pediat. Res. 6: 563-575 (1972)

Cell biology

Golgi apparatus

Hurler's syndrome lysosome mucopolysaccharide phagocytosis

\title{
An Ultrastructural Comparison of Normal and Hurler Syndrome Dermal Fibroblasts
}

\author{
Gary W. Conrad ${ }^{[52]}$, Debra Sherman, and Albert Dorfman \\ Departments of Pediatrics and Biochemistry, La Rabida-University of Chicago Institute and the Joseph P. Kennedy, Jr., \\ Mental Retardation Research Center, University of Chicago, Chicago, Illinois, USA
}

\begin{abstract}
Extract
Cultured skin fibroblasts from normal individuals and patients with Hurler's syndrome were examined by electron microscopy at different phases of the culture cycle at 7,21 , and 35 days after retrypsinization and inoculation. Cultures were incubated for $0.5-24$ hr with colloidal gold, incubated to localize acid phosphatase and aryl sulfatase B activities histochemically, and assayed for these enzymes biochemically. Both types of fibroblasts grew to saturation densities of $4 \times 10^{6}$ cells $/$ dish.

The large vacuoles seen in the cytoplasm of Hurler fibroblasts appeared to be secondary lysosomes, for they accumulated particles of colloidal gold and contained myelin-like figures. They seemed to derive from the Golgi membranes which tended to show a higher frequency of dilated cisternae than those of normal cells. Specific activities of the assayed enzymes in normal and Hurler cells were equal. In normal cells, acid phosphatase was demonstrated in the same vacuoles which accumulated colloidal gold.
\end{abstract}

\section{Speculation}

The large vacuoles of Hurler fibroblasts appear to be secondary lysosomes and to derive from the Golgi apparatus rather than from the rough endoplasmic reticulum.

\section{Introduction}

Hurler's syndrome is an autosomal recessive connective tissue disease in which large amounts of dermatan sulfate and heparan sulfate are stored in tissues and excreted in urine $[7,13,14,32,35,36]$. Cultured dermal fibroblasts from Hurler patients express this mutant genotype by accumulating excessive quantities of dermatan sulfate intracellularly [32, 33]. Although considerable data suggest that Hurler fibroblasts are defective in degradation of intracellular mucopolysaccharides $[19,20]$, absence of a specific glycosidase has not been demonstrated in Hurler tissues and cultured fibroblasts although lower concentrations of L-iduronidase have been reported in cultured Hurler fibroblasts
[31]. Concentrations of $\beta$-galactosidase in fibroblasts have been reported to be normal [5] or decreased [18]. Tissue storage and urinary excretion of mucopolysaccharides by Hurler patients have recently been reviewed $[15,37]$.

A common abnormality that is observed in cultured fibroblasts from patients with several diverse and seemingly unrelated diseases is a marked intracellular accumulation of acid mucopolysaccharides, as is evidenced by toluidine blue metachromasia in the cytoplasm and by biochemical analysis. In addition to mucopolysaccharidoses, in which abnormalities of polysaccharide metabolism seem fundamental to the disease process, disorders of lipid storage, cystic fibrosis, and muscular 
dystrophy also show metachromasia in cultured dermal fibroblasts [33].

Electron microscopy of tissue specimens from Hurler liver [39], skin [12], brain [29], and heart [28], has revealed large cytoplasmic vacuoles, presumably lysosomes, that are thought to contain partially degraded polysaccharide. It remains important, however, to study dermal fibroblasts because of their role in biosynthesis of polysaccharide. In addition, the observation of polysaccharide accumulation in dermal fibroblasts from patients with unrelated diseases suggests that a phenomenon of broad biologic significance may be operating in fibroblasts.

\section{Materials and Methods}

\section{Cell Culture}

Specimens of skin were obtained from three patients with Hurler's syndrome and from three normal control subjects by 3-mm punch biopsies from the deltoid region [47]. Fibroblast cultures established from such samples were grown under $10 \% \mathrm{CO}_{2}-90 \%$ air on 60 . $\mathrm{mm}$ plastic petri dishes [41] in modified Eagle's medium which contained $50 \mathrm{~mm} \mathrm{NaHCO}{ }_{3}, 0.57 \mathrm{~mm}$ ascorbic acid, and $10 \%$ fetal calf serum. Cells were occasionally frozen in GIB medium [42], which contained $20 \%$ fetal calf serum, $10 \%$ calf serum, and $10 \%$ dimethylsulfoxide, and were then stored in liquid nitrogen. Cells from second to eighth transfer cultures were inoculated with $3 \times 10^{5}$ cells/dish and fixed for electron microscopy during different phases of the culture cycle. Cell numbers were determined in hemocytometer counting chambers. Cells grown on cover slips were stained with toluidine blue to monitor cytoplasmic metachromasia [33].

Chromosome analysis showed normal diploid karyotypes. Several independent assays for pleural pneumococcal-like organisms (PPLO) were negative. No forms resembling PPLO or virus were seen by electron microscopy in either type of fibroblast.

\section{Phagocytosis}

Cells were incubated for varying lengths of time in nutrient medium containing colloidal gold [43]. One milliliter gold suspension was diluted with $5 \mathrm{ml} \mathrm{nu-}$ trient medium for use on cells. Addition of colloidal gold suspension directly to cells in conditioned medium followed by incubation gave results which were comparable to a procedure of complete removal of nutrient medium followed by replacement with fresh, unconditioned medium containing gold. After incubation, cells were fixed for electron microscopy with glutaraldehyde and osmium tetroxide as described below.

\section{Electron Microscopy}

Fibroblasts were fixed at $4^{\circ}$ for $15 \mathrm{~min}$ in situ with $3 \%$ glutaraldehyde in $0.08 \mathrm{~m}$ sodium cacodylate buffer (pH 7.2) that contained $109.8 \mathrm{~mm} \mathrm{NaCl}, 5.36 \mathrm{~mm} \mathrm{KCl}$, $0.88 \mathrm{mM} \mathrm{MgSO}_{4}$, and $2.39 \mathrm{~mm} \mathrm{CaCl}_{2}$. The cell layer was rinsed three times with cold cacodylate buffer and treated in situ with $1 \%$ osmium tetroxide in the same buffer for $60 \mathrm{~min}$ at $4^{\circ}$. The cells were then removed from the plastic substratum with a rubber policeman and immobilized in random orientations in an agar gel [24]. Fragments of such gels were occasionally stained en bloc at $4^{\circ}$ for $18 \mathrm{hr}$ with $2 \%$ aqueous uranyl acetate. Gels were dehydrated in ethanol and embedded in Vestopal W. Blocks were sectioned with glass knives on an ultramicrotome [44]. Silver sections, mounted on carbon-coated Formvar films on copper grids, were stained with aqueous uranyl acetate alone, or they were doubly stained with uranyl acetate followed by basic lead citrate [38]. Sections were examined in a Siemens 101, or occasionally a Siemens 1A, electron microscope [45]. Each microscope was equipped with a liquid nitrogen decontamination device, single crystal pointed filaments, and $50 \mu$-objective apertures at accelerating voltages of 80 or $100 \mathrm{kv}$.

\section{Enzyme Activity}

In Situ. Aryl sulfatase B activity was localized by light and electron microscopy. Cells, attached to petri dishes and cover slips, were fixed with glutaraldehyde as described above and then incubated with $p$-nitrocatechol sulfate for 90 and $135 \mathrm{~min}$ for electron microscopy or 90,135 , and $180 \mathrm{~min}$ for light microscopy [1, 22]. Substrate was omitted from control incubations.

Acid phosphatase activity was localized by light microscopy after fixation in calcium-formol solution [2], and by electron microscopy after the glutaraldehyde fixation step described above. The azo dye technique [2] (with naphthol AS-TR phosphate as substrate) and the Gomori procedure [2] (with $\beta$-glycerophosphate as substrate) were used to demonstrate activity by light microscopy after 30-, 60-, and 90-min incubation. The same Gomori procedure [34] was also employed to demonstrate activity by electron microscopy after 12 and 30-min incubation. Substrate was omitted from control incubations.

Cell Sonicates. Approximately $4 \times 10^{7}$ normal and 
Hurler fibroblasts (21 days, late logarithmic growth phase) were scraped from dishes with rubber policemen, centrifuged, and rinsed once in phosphate-buffered saline. Pellets were resuspended in 2 volumes of $0.2 \mathrm{M}$ sucrose, sonicated for $2 \mathrm{~min}$ at $0^{\circ}$ [46], and assayed for enzyme activity in the presence of $0.1 \%$ Triton X-100. Acid phosphatase and aryl sulfatase B activities were assayed by the method of Bowers et al. [6], and protein was measured by the method of Lowry et al. [30].

\section{Results}

Control experiments indicate that the fixation method satisfactorily preserved cellular fine structure. Other methods of fixation were less satisfactory; they included Karnovsky's fixative [26], glutaraldehyde in nutrient medium or in cacodylate buffer with glucose, and mixtures of glutaraldehyde and osmium tetroxide [24]. None of these methods obscured the salient differences between normal and Hurler fibroblasts.

\section{Effect of Time in Culture on Cellular Ultrastructure}

In no stage of the culture cycle studied did the cytoplasm of normal cells display metachromatic granules, but that of Hurler fibroblasts always contained such structures. Increase in cell number with time for both types of cells is shown in Figure 1. Hurler fibroblasts grew at a slightly slower rate than did normal cells, but they reached a cell density during the saturation phase which was equal to that of normal fibroblasts. The ultrastructure of cells in the logarithmic phase of growth ( 7 and 21 days) was indistinguishable from that of cells in the saturation phase (35 days). Cultures older than 49 days were not examined. We observed no drastic changes in the proportions of free and attached ribosomes during the different phases of growth. These observations thus seem to contradict those of Goldberg and Green [21] of mouse fibroblasts in vitro and those of Comings and Okada [9] of trypsinized human fibroblasts in vitro; in both studies, less rough endoplasmic reticulum was found in logarithmic phase than in saturation phase.

Normal fibroblasts could be consistently distinguished from Hurler cells during any stage of the culture cycle by the criteria of vacuole number and Golgi morphology (described below). Furthermore, the same conclusions were valid when cells from third to ninth transfer cultures were used as inoculum for a culture cycle experiment. Senescent, nongrowing cells, i.e., older than the ninth transfer, were not examined. In-

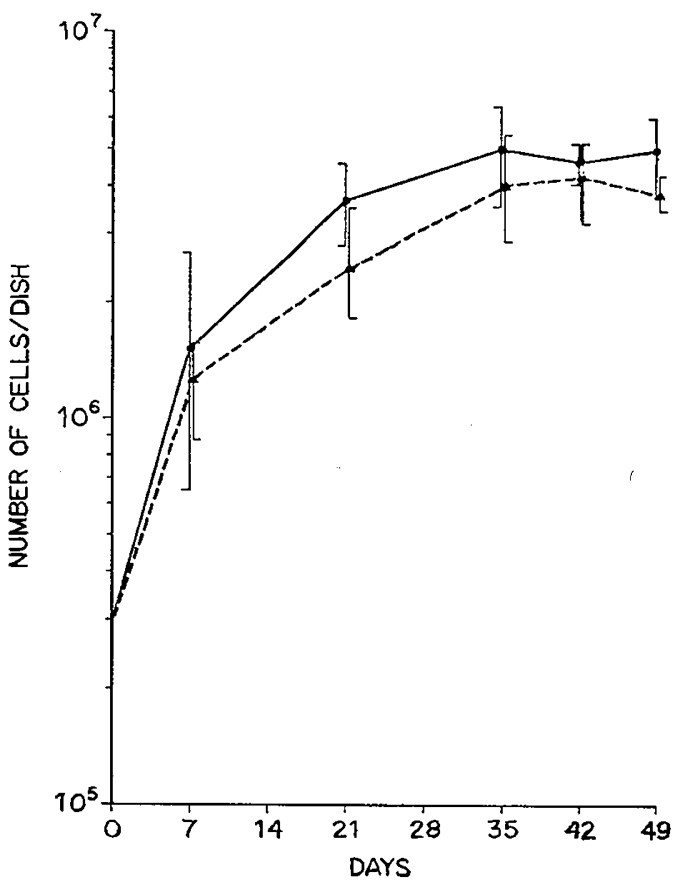

Fig. 1. Increase in numbers of normal and Hurler fibroblasts with time in culture. Solid line represents normal cells; dotted line signifies Hurler cells. Length of vertical bars indicates the range of cell numbers for each point. Points on the normal graph represent averages of combined data from seven separate experiments with cells from normal patients $A, B$, and $C$; Hurler graph is derived from seven separate experiments with cells from Hurler patients $D$ and $E$.

asmuch as the transfer number of a culture is roughly proportional to the total number of mean population doublings in vitro, we conclude that differences in cell density, time in vitro since the last trypsinization, and the number of population doublings in vitro were not associated with alterations in cellular ultrastructure within the limits of these experiments. The greatest differences observed in cell morphology, therefore, derived from the Hurler mutation.

\section{Morphologic Differences between Normal and Hurler Fibroblasts}

The cytoplasm of typical normal fibroblasts (Fig. 2) contained only about one-tenth as many vacuoles as did typical Hurler fibroblasts (Fig. 3). (The term "vacuole" is used here in a purely descriptive sense and signifies a generally spherical area limited by a single unit membrane. This term implies no association or lack of association with lysosomal activity.) However, approximately $1-5 \%$ of normal fibroblasts had as many large vacuoles as did Hurler cells (Fig. 2). With 


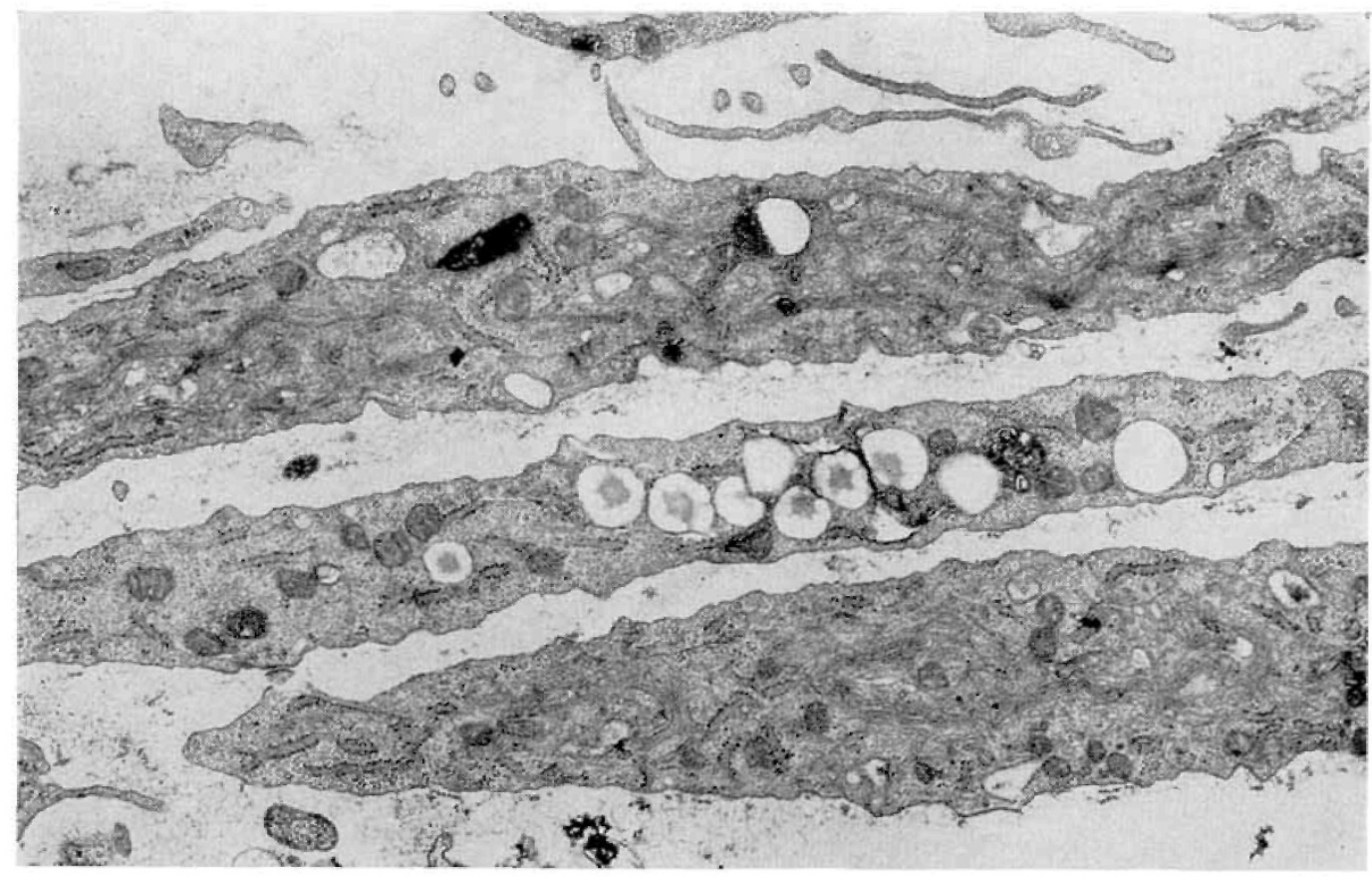

Fig. 2. Three normal fibroblasts. Middle cell contains an unusually large number of vacuoles. Such cells constitute approximately $1-5 \%$ of the total cell population, but they are consistently present. The other two cells display extensive arrays of Golgi lamellae and some rough endoplasmic reticulum, but few vacuoles. Patient $C$, eighth transfer, 7-day culture. $\times 15,470$.

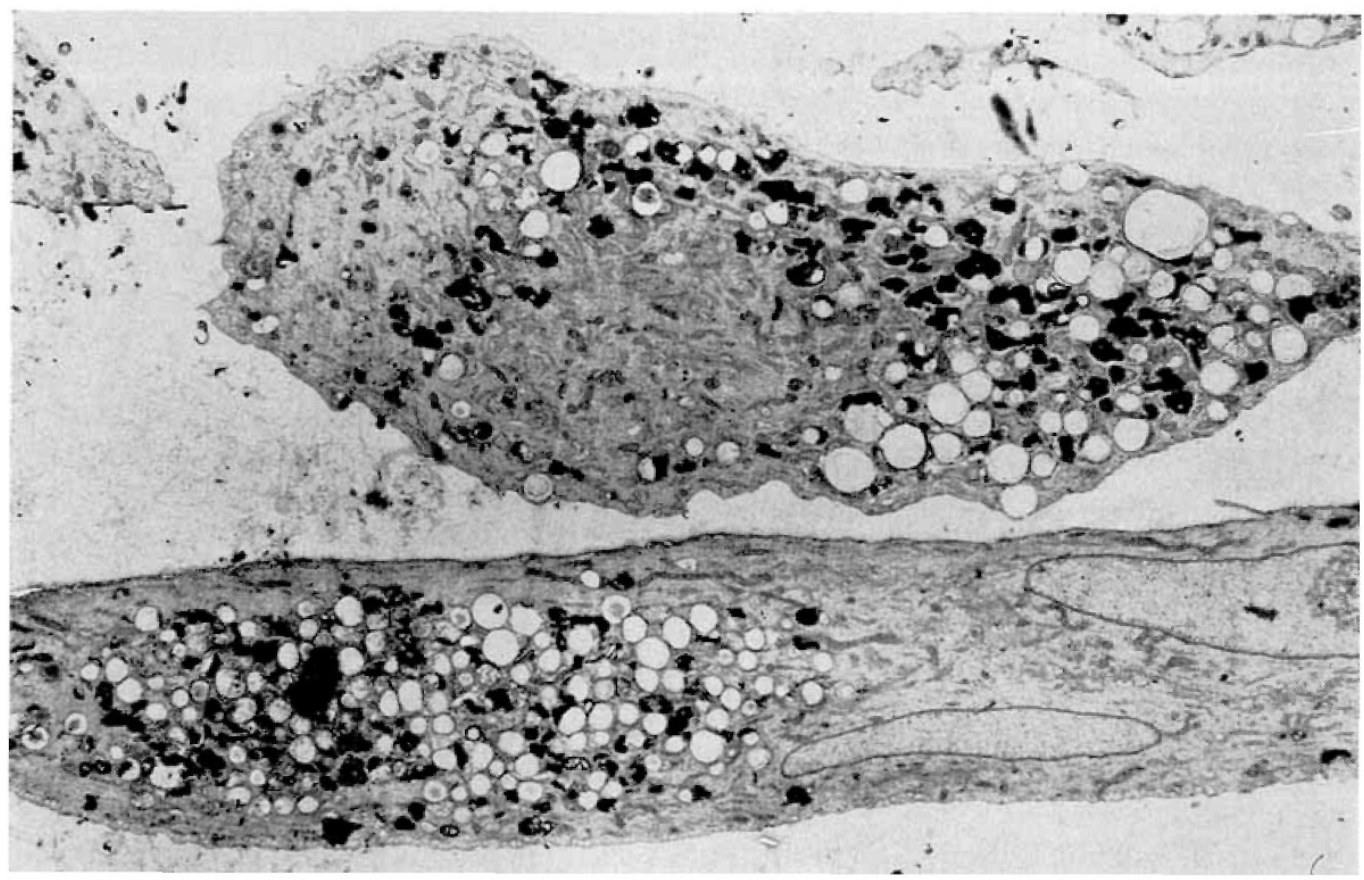

Fig. 3. Two Hurler fibroblasts. Cytoplasm of each cell contains large numbers of vacuoles which enclose materials of varying electron density. Clear vacuoles are probably empty as a result of leaching during fixation. Residual bodies are abundant. Patient $F$, third transfer, 21 day culture. $\times 4,625$. 
the fixation techniques used, many vacuoles in Hurler cells appeared empty except for a fine reticular network in the central space and some heterogeneous material along one side. Other vacuoles, however, contained material of increasing degrees of electron density evenly distributed as a granular matrix (Fig. 4). Cytoplasmic vacuoles present in normal fibroblasts were few in number, but they were comparable to those of Hurler fibroblasts in size, distribution, variety of contents, and morphology.

Residual bodies, showing myelin-like arrangements, were present in many of the vacuoles of both normal and Hurler cells. Their total number per cell seemed to be greater in Hurler cells, however, perhaps in proportion to the greater number of vacuoles. After uranyl acetate staining, the bodies were generally opaque, but, with thinner sections and higher accelerating voltages, the typical layering of the component membranes was evident (Fig. 5). Residual bodies were enclosed in unit membrane-limited vacuoles. None was observed outside cells or in association with the plasma membrane. The shape of these structures was variable. Frequently they consisted of spheres of concentric membranous layers arranged around an electron-transparent core, but they were also present within vacuoles as stacks of membranous lamellae apposed to the vacuolar membrane (Fig. 5).

In Hurler fibroblasts there seemed to be a connection between the Golgi apparatus and enlarged cytoplasmic sacs (Fig. 6, arrows). Although the Golgi apparatus in normal fibroblasts seemed to give rise mainly to small vesicles (Fig. 7), that of Hurler fibroblasts was more frequently associated with dilated sacs of smooth endoplasmic reticulum (Fig. 6). These dilatations occurred more frequently at the tips or edges of the Golgi lamellae than in the central regions. To determine the significance of this observation, an attempt was made to compare the numbers of normal cells and Hurler cells which had dilated Golgi sacs. Only photomicrographs showing definitive Golgi areas were scored, and no two photomicrographs were of the same cell. Nondilated Golgi lamellae in normal cells are seen in Figures 2 and 7. Nondilated Golgi lamellae in Hurler cells are seen in Figure 4 (inset). Of 66 photomicrographs of normal cells, only $3(5 \%)$ showed dilated Golgi lamellae. However, of 51 similar photomicrographs of Hurler cells, 21 (41\%) showed dilated Golgi lamellae.

The Golgi apparatus in both normal and Hurler cells occasionally displayed a lamellate, cuplike morphology in cross section (Fig. 6, inset) (see also [17]), but more frequently the lamellar sacs were spread out in flattened arrays (Figs. 2 and 4, inset). In such cases it was difficult to distinguish the secretory face of the Golgi apparatus from the forming face, and we could not discern with which face the dilated sacs were associated.

In the region of the Golgi apparatus of the Hurler cells, there was, in addition to the dilated sacs described above, a variety of sacs or vacuoles containing material of varied granularity and electron density. The less electron-dense vacuoles seemed to be more frequently spherical. These observations may indicate that at least some of the characteristic, large cytoplasmic vacuoles of Hurler cells arise in the area of the Golgi apparatus.

\section{Enzyme Activity}

In Situ. To determine whether any of the vacuoles observed in normal cells were lysosomes, an attempt was made to demonstrate acid hydrolase activity in situ. By the techniques of ultrastructural histochemistry described previously, aryl sulfatase $\mathrm{B}$ activity was localized in some, but not all, of the infrequent, large vacuoles and residual bodies of normal cells. By light microscopy, normal fibroblasts contained reaction product at all incubation times. Controls had no reaction product.

Acid phosphatase activity was limited to some, but not all, of the infrequent, large vacuoles and residual bodies of normal cells. By both the Gomori technique and the azo dye procedure, light microscopy showed that normal fibroblasts contained reaction product at all incubation times. Controls for all techniques had no reaction product.

Neither of the above enzymes could be demonstrated histochemically in Hurler vacuoles.

Cell Sonicates. Aryl sulfatase B and acid phosphatase in total cell sonicates were assayed biochemically in order to quantitate the relative levels of enzyme activities in normal and Hurler fibroblasts. These assays indicated that activities of both enzymes were comparable in the two types of fibroblasts (Table I).

\section{Phagocytosis}

Normal and Hurler fibroblasts were incubated in nutrient medium containing colloidal gold. Particles of gold were observed in both types of cells after 12-hr incubation and were readily evident after $24 \mathrm{hr}$. No particles were observed after incubations of $30 \mathrm{~min}, 1$ $\mathrm{hr}$, and $2 \mathrm{hr}$. Colloidal gold did not seem to be toxic 


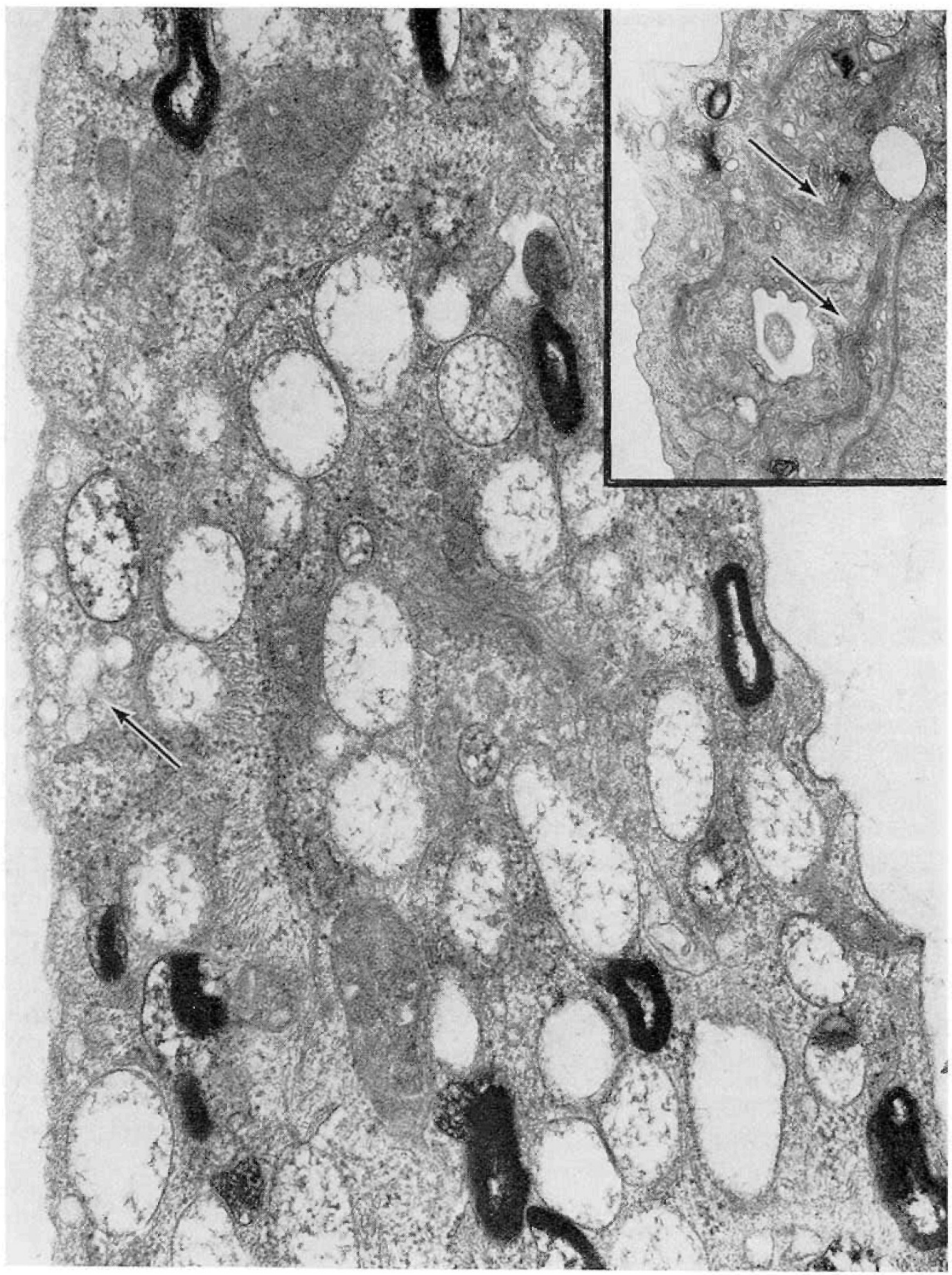

Fig. 4. Cytoplasm of a Hurler fibroblast. The cytoplasm contains large vacuoles, some of which surround densely stained residual bodies (Fig. 5). Vacuolar membranes are of the unit membrane type. Small, smooth-surfaced vesicles are seen near the plasma membrane (arrow) and also near the flattened Golgi apparatus (inset, arrows). Inset, from another cell, also shows a cilium in cross section. Patient $D$, seventh transfer, 7-day culture. $\times 42,700$. Inset: $\times 21,000$. 


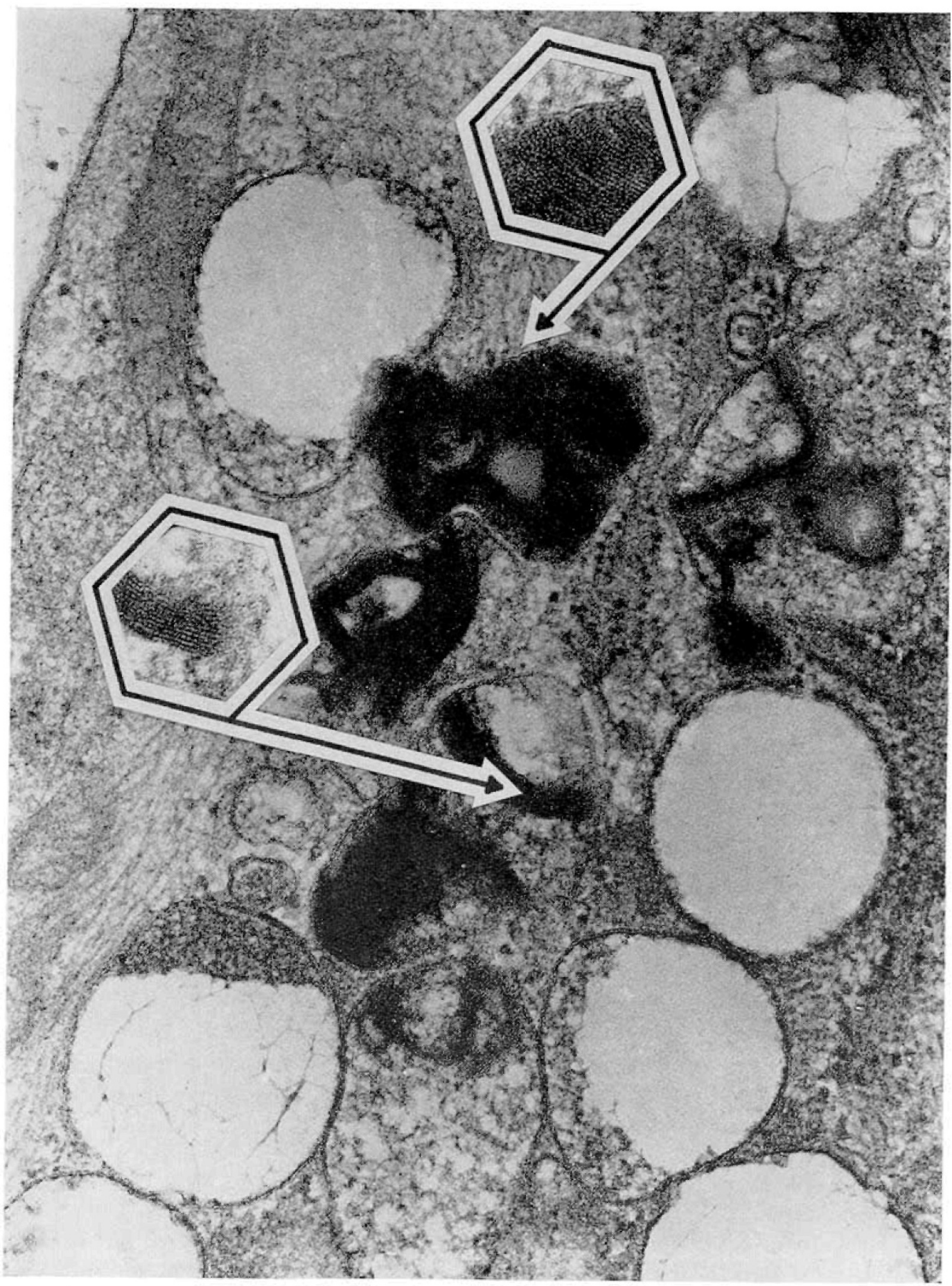

Fig. 5. Cytoplasm of a Hurler fibroblast. $A$ representative array of vacuoles and their inclusions is shown. Vacuoles may be nearly empty, show a reticular network with material aggregated on one side, or contain residual bodies which have a myelin-like layering of membrane material. Upper inset shows lamellar associations at the vacuolar membrane. Lower inset shows lamellae in association with amorphous material within vacuole. Patient $D$, third transfer, 21 -day culture. $\times 65,600$. Insets: $\times 150,000$. 


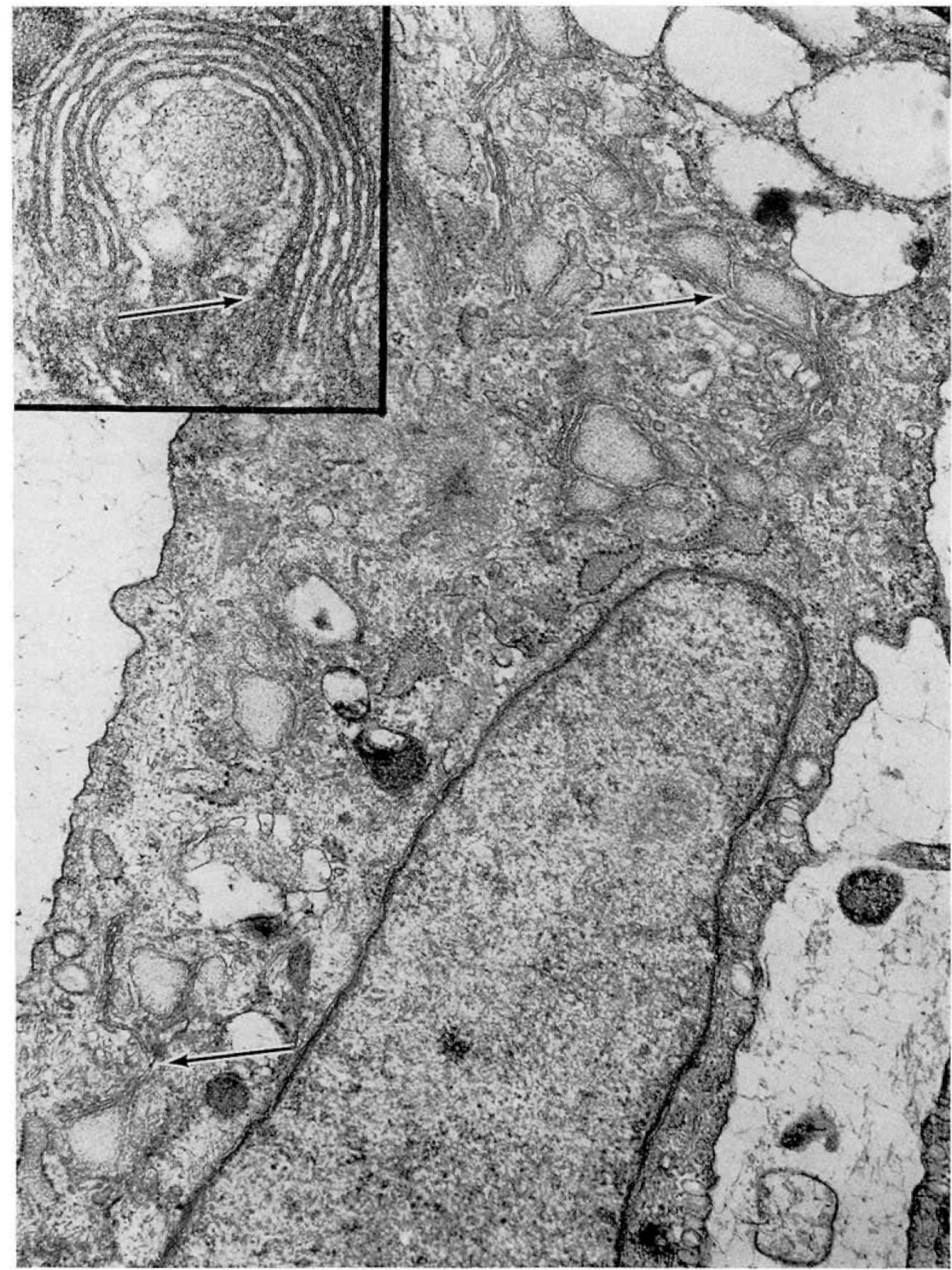

Fig. 6. A Hurler fibroblast showing the Golgi region. Tips (or edges) of the Golgi lamellae are dilated to form large sacs containing amorphous material equal or lower in density than that in the cisternae of the rough endoplasmic reticulum. Such sacs appear to be directly connected to the Golgi lamellae (arrows). The Golgi area also contains smooth-surfaced vesicles similar to those found attached to the plasma membrane (lower right). Inset, from another cell, shows a cross section of the Golgi apparatus associated with an expanded sac. Patient D, seventh transfer, 35-day culture. $\times 28,500$. Inset: Patient $D$, fourth transfer, 21-day culture. $\times 60,000$. 


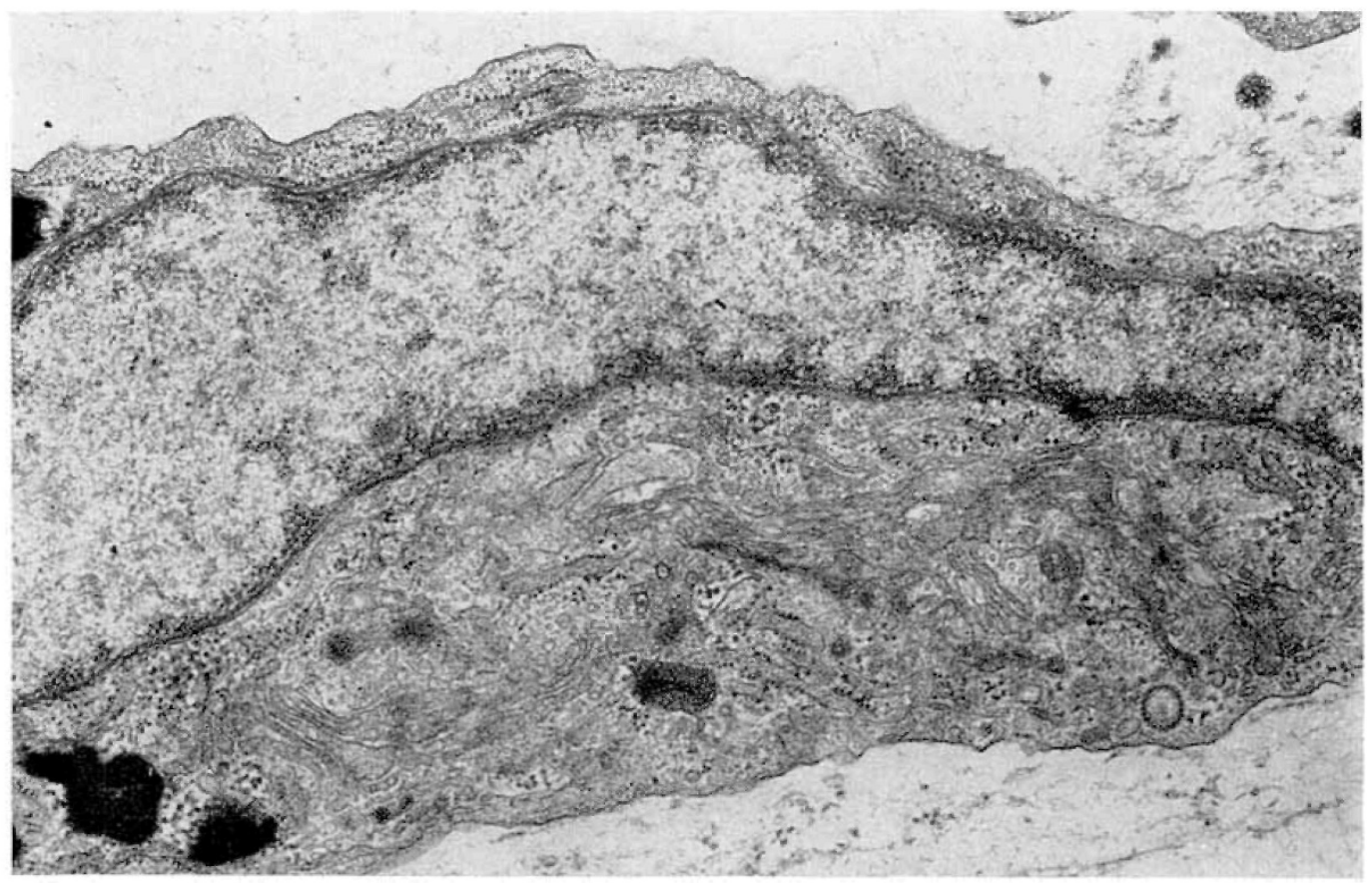

Fig. 7. A normal fibroblast. Golgi apparatus is arranged in flattened lamellae which appear to be giving rise to smooth-surfaced vesicles similar to ones seen attached to the plasma membrane. Only one coated vesicle is seen. Golgi lamellae are not associated with large sacs. Patient B, seventh transfer, 35-day culture. $\times 26,250$.

for at least 4 days of incubation. A Hurler fibroblast incubated for $24 \mathrm{hr}$ with colloidal gold is shown in Figure 8. Gold particles were contained within some, but not all, of the vacuoles and residual bodies in both normal and Hurler cells. They were never seen in the small, smooth-surfaced vesicles described below, but were seen in one case within a coated vesicle.

Because not all vacuoles showed enzyme activity or contained gold particles, it was possible that two separate populations of vacuoles were present. Fibroblasts were incubated with gold for $24 \mathrm{hr}$, fixed, and then incubated for acid phosphatase activity as previously described. These experiments indicated that gold particles could be sequestered in the same vacuoles in which acid phosphatase activity was present. Thus, material ingested by phagocytosis, in normal fibroblasts at least, may be transported to active secondary lysosomes. This experiment was not done with Hurler fibroblasts because of the difficulty in demonstrating enzyme activity histochemically in these cells. The inability to demonstrate acid phosphatase and aryl sulfatase B activities histochemically in Hurler fibroblasts, under the same conditions which allowed such demonstration in normal fibroblasts, is not understood at present.
Table I. Acid phosphatase and aryl sulfatase B activities in sonicates of normal and Hurler fibroblasts

\begin{tabular}{ccc}
\hline Fibroblasts & Acid phosphatase $^{1}$ & Aryl sulfatase B2 $^{2}$ \\
\hline Normal & 1.80 & 0.48 \\
Hurler & 1.54 & 0.47 \\
\hline
\end{tabular}

${ }^{1}$ Micromoles phosphate released per milligram protein per hour.

${ }^{2}$ Micromoles 4-nitrocathechol released per milligram protein per hour.

\section{Cytoplasmic Structures Common to Normal and Hur- ler Fibroblasts}

Cytoplasmic filaments were regularly seen in the ground substance of both types of fibroblasts. Microtubules were observed only in association with the basal bodies of cilia. Cilia were regularly seen in both types of cells although their frequency was not measured.

Along the plasma membrane of both normal and Hurler cells were smooth-surfaced and coated vesicles (Figs. 4 and 7). The number of coated vesicles observed was only approximately $5 \%$ of the number of smoothsurfaced vesicles. Smooth-surfaced vesicles were somewhat ellipsoid in shape when seen in longitudinal section, and they appeared to be attached to the plasma 


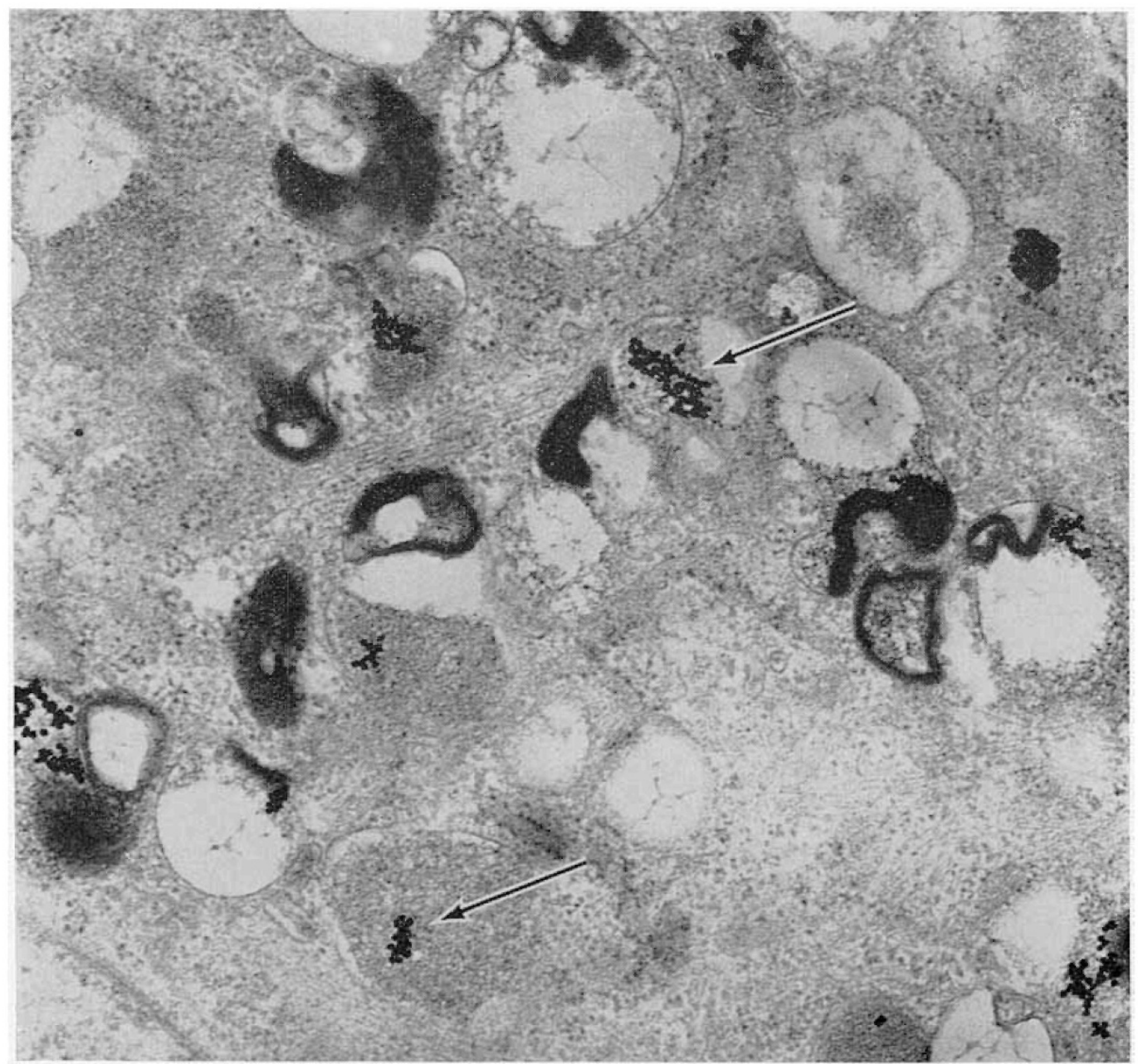

Fig. 8. Hurler fibroblast. Cells were incubated for $24 \mathrm{hr}$ in medium containing colloidal gold. Gold particles (arrows) are confined to the interiors of vacuoles and frequently were associated with residual bodies. Companion cultures of normal fibroblasts sequestered gold in similar types of vacuoles. Patient $D$, fifth transfer, 21 -day culture. $\times 36,700$.

membrane in large numbers in such a way that the vesicle interiors were continuous with the extracellular space. When seen in tangential section, they were arranged in clusters. We did not observe structures which suggested extensive fusion among these smoothsurfaced vesicles or with larger vesicles. Vesicles similar to the smooth vesicles at the plasma membrane were seen in association with the Golgi apparatus. The coated and the smooth-surfaced type vesicles seen in Hurler fibroblasts appeared indistinguishable from those in normal fibroblasts.

\section{Discussion}

Recently published results of three ultrastructural studies of Hurler or Hunter fibroblasts present certain inconsistencies. Bartman and Blanc [3] believe that the large cytoplasmic vacuoles observed in Hurler fibroblasts may arise directly from dilated rough endoplasmic reticulum. No mention was made of the Golgi apparatus. DeCloux and Friederici [12] examined Hurler dermal fibroblasts in biopsies of skin but could not determine the source of the large cytoplasmic vacuoles. They postulated that the vacuoles probably originated from the Golgi apparatus. Because, in this work, dilated rough endoplasmic reticulum in the region of the Golgi apparatus occurred in both normal and Hurler cells, this phenomenon is probably not characteristic of the Hurler phenotype. Abnormalities of mitochondria reported in Hurler [12] and Hunter [16] fibroblasts were not observed by us in Hurler fibroblasts.

Data derived from toluidine blue staining $[10,11]$ and from biochemical analyses [32, 33] demonstrate 
that dermal fibroblasts derived from Hurler individuals accumulate polysaccharides in their cytoplasm. Three possible explanations have been proposed: $(l)$ oversynthesis [32], (2) faulty degradation [19], and (3) defective secretion [32]. On the basis of similarity to other storage diseases and other evidence, faulty degradation seems to be the most likely explanation, but it has not yet been finally established [14].

The presence of large numbers of single membranelimited vacuoles in the cytoplasm of Hurler cells indicates a similarity to other storage diseases $[4,23,25$, 40]. The fact that the interiors of these vacuoles are generally of low electron density suggests that material originally present may have been lost during fixation. Removal of protein from the acid mucopolysaccharide-protein complexes by lysosomal proteases would account for these findings and would be consistent with the report of Fratantoni et al. [19] that the intracellular mucopolysaccharide is of lower molecular weight than that excreted into the medium.

The idea that the spherical structures of normal and Hurler cells may indeed be secondary lysosomes is supported by their uptake of gold particles and the presence of myelin-like figures. The presumed secondary lysosomes of Hurler fibroblasts seem to differ from the demonstrated secondary lysosomes of normal cells in number, but not in range of sizes or morphologies. Histochemical experiments with Hurler fibroblasts showed an almost complete absence of both acid phosphatase and aryl sulfatase $B$ activities. Inasmuch as the biochemical data for these enzymes (Table I) suggest equal activities in normal and Hurler cells, further study is required to explain the anomalous histochemical data for Hurler cells.

Of possible interest in our studies was the observation of dilated Golgi cisternae in Hurler fibroblasts. Their relation to the large cytoplasmic vacuoles, i.e., secondary lysosomes, is of importance. If one considers the origin of the increased number of large, secondary lysosomes in Hurler fibroblasts, several possibilities are apparent.

1. Both normal and Hurler fibroblasts not only synthesize protein-polysaccharide but they also engage in phagocytosis. To some degree, therefore, they ingest their own extracellular secretion products. If Hurler fibroblasts ingested such material more quickly than it could be degraded, polysaccharide accumulation might result. If the rates of phagocytosis were approximately equal, accumulation would occur if Hurler lysosomes were defective. The rates of phagocytosis and pinocytosis in the two types of fibroblasts should be compared.
2. Fratantoni et al. [19] suggested that a portion of the mucopolysaccharide-protein complex normally synthesized is destined for intracellular degradation. It is proposed that a faulty degradation mechanism is responsible for the accumulation of acid mucopolysaccharides in Hurler's disease. This hypothesis, like the first one, requires the demonstration of a defective lysosomal enzyme in Hurler cells.

It has been demonstrated that cultured fibroblasts secrete protein factors, perhaps lysosomal enzymes, directly into the nutrient medium. These factors, upon uptake by or contact with fibroblasts of a different genotype, can lead to cessation of polysaccharide accumulation in one or both populations of cells, a phenomenon of cellular complementation $[8,20,27]$. Our study shows that large molecular weight material such as colloidal gold or protein factors can be taken into Hurler cells and sequestered in the secondary lysosomes, the presumed site of polysaccharide accumulation. If the protein factors are indeed lysosomal enzymes, the way in which they cause cessation of polysaccharide accumulation is direct and obvious.

3. Polysaccharide might accumulate in Hurler fibroblasts if phagocytosis, biosynthesis, and turnover were normal, while the process of secretion was faulty. Two lines of evidence suggest that faulty secretion is not involved in the Hurler syndrome. First, kinetic data suggest that the rate of excretion from Hurlex cells is the same as that in normal cells. Second, the alteration or absence of a single lysosomal enzyme, e.g., L-iduronidase, would constitute a simpler explanation of polysaccharide accumulation. Nevertheless, the data collected in the present study suggest greater dilatation of the Golgi sacs in Hurler than in normal cells, which is perhaps simply a reflection of their involvement in the production of the large lysosomes filled with polysaccharide.

Although conclusive evidence awaits detailed analysis of both purified lysosomes and Golgi vesicles, the dilatation of the Golgi cisternae and the aberrant histochemical characteristics of the Hurler lysosomes suggested by our study are factors to be considered in the pathogenesis of Hurler's syndrome.

\section{Summary}

The ultrastructures of dermal fibroblasts from normal humans and from patients with Hurler's syndrome were compared. Differences in cell density, time in vitro following the last trypsinization, and the number of population doublings in vitro were not associated 
with alterations in ultrastructure. The greatest differences in cellular ultrastructure arose from the Hurler mutation. Hurler cells consistently contained granules which stained metachromatically with toluidine blue, but normal cells rarely contained such granules. At all stages of the culture cycle, Hurler fibroblasts displayed many more large cytoplasmic vacuoles than did normal cells.

Aryl sulfatase $\mathbf{B}$ activity and acid phosphatase activity were demonstrated histochemically in the infrequent, large cytoplasmic vacuoles and residual bodies of normal fibroblasts, but both activities were absent from histochemical preparations of Hurler cells. Enzymic analysis of both normal and Hurler fibroblasts, however, showed that aryl sulfatase B and acid phosphatase had comparable activities per milligram protein. After 12-hr incubation with colloidal gold, particles were taken into normal and Hurler fibroblasts by phagocytosis and sequestered in the vacuoles and residual bodies. We conclude that the vacuoles seen in normal and Hurler fibroblasts are secondary lysosomes because they contain material of both extracellular and intracellular origin.

In addition to the large cytoplasmic vacuoles, Hurler fibroblasts also appeared to have more dilated cisternae of the Golgi apparatus than did normal cells. In both types of cells, the Golgi apparatus was associated with many small, smooth-surfaced vesicles, but in Hurler cells it also appeared to give rise to at least some of the large vacuoles.

\section{References and Notes}

1. Bainton, D. F., And Farquhar, M. G.: Segregation and packaging of granule enzymes in eosinophilic leukocytes. J. Cell Biol., 45: 54 (1970).

2. Barka, T., ANd Anderson, P. J.: Histochemistry: Theory, Practice, and Bibliography, p. 238. (Harper and Row, New York, 1963).

3. Bartman, J., AND Blanc, W. A.: Fibroblast cultures in Hurler's and Hunter's syndromes. An ultrastructural study. Arch. Pathol., 89: 279 (1970).

4. Baudhuin, P., Hers, H. G., and Loeb, H.: An electron microscopic and biochemical study of Type II glycogenosis. Lab. Invest., 13: 1139 (1964).

5. Benson, P. F., Bowser-Riley, F., and Giannelli, F.: $\beta$-Galactosidases in fibroblasts: Hurler and Sanfillippo syndromes. New Engl. J. Med., 283: 999 (1970).

6. Bowers, W. E., Finkenstaedt, J. T., and DeDuve, C.: Lysosomes in lymphoid tissue. I. The measurement of hydrolytic activities in whole homogenates. J. Cell Biol., 32: 325 (1967).

7. Brown, D. H.: Tissue storage of mucopolysaccharides in Hurler-Pfaundler's disease. Proc. Nat. Acad. Sci. U. S. A., 43: 783 (1957).

8. Gantz, M., Chrambach, A., and Neufeld, E. F.: Characterization of the factor deficient in the Hunter syndrome by polyacrylamide gel electrophoresis. Biochem. Biophys. Res. Commun., 39: 936 (1970).

9. Comings, D. E., AND OKadA, T. A.: Electron microscopy of human fibroblasts in tissue culture during logarithmic and confluent stages of growth. Exp. Cell Res., 61: 295 (1970).

10. Danes, B. S., and Bearn, A. G.: Hurler's syndrome. A genetic study in cell culture. J. Exp. Med., 123: 1 (1967).

11. Danes, B. S., And Bearn, A. G.: Hurler's syndrome. A genetic study of clones in cell culture with particular reference to the Lyon hypothesis. J. Exp. Med., 126: 509 (1967).

12. DeCloux, R. J., ANd Friedericr, H. H. R.: Ultrastructural studies of the skin in Hurler's syndrome. Arch. Pathol., 88: 350 (1969).

13. Dorfman, A., And Lorincz, A. E.: Occurrence of urinary acid mucopolysaccharides in the Hurler syndrome. Proc. Nat. Acad. Sci. U. S. A., 43: 443 (1957).

14. Dorfman, A., and Matalon, R.: The Hurler and Hunter syndromes. Amer. J. Med., 47: 691 (1969).

15. Dorfman, A., and Matalon, R.: The mucopolysaccharidoses. In: J. B. Stanbury, J. B. Wyngaarden, and D. S. Frederickson: The Metabolic Basis of Inherited Disease, Ed. 3. (The Blakiston Division, McGraw-Hill, New York, 1971).

16. Ducketr, S., Christian, J. C., Thompson, J. N., and Drew, A. L.: The ultrastructure of metachromatic bodies in cultured fibroblasts in Hunter's syndrome. Develop. Med. Child Neuxol., 11: 764 (1969).

17. Flickinger, C. J.: The development of Golgi complexes and their dependence upon the nucleus in amebae. J. Cell Biol., 43: 250 (1969).

18. Fluharty, A. L., Porter, M. T., Lassila, E. L., Trammell, J., Carrel, R. E., and Kihara, H.: Acid glycosidases in mucopolysaccharidoses' fibroblasts. Biochem. Med., 4: 110 (1970).

19. Fratantoni, J. C., Hall, C. W., and Neufeld, E. F.: The defect in Hurler's and Hunter's syndromes: faulty degradation of mucopolysaccharide. Proc. Nat. Acad. Sci. U. S. A., 60: 699 (1968).

20. Fratantoni, J. C., Hall, C. W., and Neufeld, E. F.: Hurler and Hunter syndrome: mutual correction of the defect in cultured fibroblasts. Science, 162: 570 (1968).

21. Goldberg, B., AND Green, H.: An analysis of collagen secretion by established mouse fibroblast lines. J. Cell Biol., 22: 227 (1964).

22. Goldrischer, S.: The cytochemical demonstration of lysosomal aryl sulfatase activity by light and electron microscopy. J. Histochem. Cytochem., 13: 520 (1965).

23. Hashimoto, K., Gross, G. B., ANd Lever, W. F.: Angiokeratoma corporis diffusum (Fabry). Histochemical and electron microscopic studies of the skin. J. Invest. Derm., 44: 119 (1965).

24. HiRSCH, J. G., AND FEDORKo, M. E.: Ultrastructure of human leukocytes after simultaneous fixation with glutaraldehyde and osmium tetroxide and "postfixation" in uranyl acetate. J. Cell Biol., 38: 615 (1968).

25. JORDAN, S. W.: Electron microscopy of Gaucher cells. Exp. Mol. Pathol., 3: 217 (1964).

26. Karnovskx, M. J.: A formaldehyde-glutaraldehyde fixative of high osmolality for use in electron microscopy. (Abstract), J. Cell Biol., 27: 137A (1965).

27. Kresse, H., Wiesmann, U., Cantz, M., Hall, C. W., and NeUfeld, E. F.: Biochemical heterogeneity of the Sanfillippo syndrome: preliminary characterization of two deficient factors. Biochem. Biophys. Res. Commun. 42: 892 (1971). 
28. Lagunoff, D., and Gritzkn, T. L.: The site of mucopolysaccharide accumulation in Hurler syndrome. Lab. Invest., 15: 1578 (1966).

29. Loeb, H., Jonniaux, G., Resibors, A., Cremer, N., Dodion, J., Tondeur, M., Gregorre, P. E., Richard, J., ANd Cieters, P.: Biochemical and ultrastructural studies in Hurler's syndrome. J. Pediat., 73: 860 (1968).

30. Lowry, O. H., Rosebrough, N. J., Farr, A. L., ANd Randall, R. J.: Protein measurement with the Folin phenol reagent. J. Biol. Chem., 193: 265 (195I).

31. Matalon, R., Cifoneldi, J. A., and Dorfman, A.: L-Iduronidase in cultured human fibroblasts and liver. Biochem. Biophys. Res. Commun., 42: 340 (197I).

32. Matalon, R., and Dorfman, A.: Hurler's syndrome: biosynthesis of acid mucopolysaccharides in tissue culture. Proc. Nat. Acad. Sci. U. S. A., 56: 1310 (1966).

33. Matalon, R., and Dorfman, A.: Acid mucopolysaccharides in cultured human fibroblasts. Lancet, $i i$ : 838 (1969).

34. Maunsbach, A. B.: Observations on the ultrastructure and acid phosphatase activity of the cytoplasmic bodies in rat kidney proximal tubule cells. J. Ultrastruct. Res., 16: 197 (1966).

35. Meyer, K., Grumbach, M. M., Linker, A., and Hoffman, P.: Excretion of sulfated mucopolysaccharides in gargoylism (Hurlex's syndrome). Proc. Soc. Exp. Biol. Med., 97: 275 (1958).

36. Meyer, K., Hoffman, P., Linker, A., Grumbach, M. M., and SAMPSON, P.: Sulfated mucopolysaccharides in urine and organs in gargoylism (Hurler's syndrome). II. Additional studies. Proc. Soc. Exp. Biol. Med., 102: 587 (1959).

37. Neufeld, E. F., and Fratantoni, J. C.: Inborn errors of mucopolysaccharide metabolism. Science, 169: 141 (1970).

38. REYNoLDs, E. S.: The use of lead citrate at high $\mathrm{pH}$ as an electron-opaque stain in electron microscopy. J. Cell Biol., 17: 208 (1963)
39. VAN Hoof, F., ANd Hers, H. G.: The ultrastructure of hepatic cells in Hurler's disease (gargoylism). C. R. Hebd. Seances Acad. Sci. (Paris), 259: 1281 (1964).

40. Volk, B. W., and Wallace, B. J.: The liver in lipidosis. An electron microscopic and histochemical study. Amer. J. Pathol., 49: 203 (1966).

41. No. 3002, Falcon Plastics, Los Angeles, Calif.

42. Grand Island Biological Company, Grand Island, N. Y.

43. Sterile colloidal gold suspension, Matheson, Coleman, and Bell, Norwood, O.

44. Porter-Blum MT-2, Ivan Sorvall, Inc., Norwalk, Conn.

45. Siemens America, New York, N. Y.

46. Branson sonifier, Branson Instrument Company, Stamford, Conn.

47. All biopsy specimens were obtained with informed consent in accordance with the Declaration of Helsinki.

48. The authors are indebted to Dr. Reuben Matalon for providing the human cell cultures on which this study was performed and for helpful discussions. They wish to thank Dr. Hewson Swift for continuing advice and for extensive use of a Siemens 1A electron microscope and ancillary facilities. The technical assistance of Mrs. Kanitha Frogner, Mrs. Harriet Martindale, and Miss Minerva Norella are gratefully acknowledged.

49. A preliminary report of this work has appeared in J. Cell Biol., 47: 39a (1970).

50. G. W. Conrad was the recipient of Public Health Service Postdoctoral Fellowship no. 5-FO2-AM-32,877-02.

51. Supported by Grants nos. AM-05996 and HD-00001 of the United States Public Health Service and Chicago and Illinois Heart Associations.

52. Requests for reprints should be addressed to: Albert DorfMAN, M.D., Department of Pediatrics, University of Chicago, Chicago, Illinois 60637 (USA).

53. Accepted for publication December 14, 1971 